\title{
Assessment of the nutritional status of bottle-fed infants and the prevalence of infections, allergy and diarrhea among bottle fed infants and its comparison with exclusively breast fed infants aged 0-6 months
}

\begin{abstract}
Title: Assessment of the Nutritional Status of Bottle-fed Infants and the prevalence of infections, allergy and diarrhea among them and its comparison with exclusively breast fed inafants aged 0-6months.

Background: For the past few years, the rate of bottle-feeding practice during the first six months of the child's life has shown a sharp increase, which necessitated to conduct a study to highlight the nutritional status of bottle-fed infants.

Objectives

To assess the nutritional status of infants who are bottle-fed aged 0-6months To assess the prevalence of infections, diarrhea and allergy among bottle-fed infants To compare the nutritional status of bottle-fed infants with exclusively breast fed infants.

Methodology: The data was collected from hospitals all over Karachi by purposive sampling. The sample size was 100 of which 50 were bottle-fed infants and 50 were exclusively breast-fed infants. The tool that was used to collect data was a questionnaire that covered different aspects including demographic, anthropometric measurements and overall health to assess the nutritional status of bottle-fed infants. A chi-square test was applied.
\end{abstract}

Result: The results of the study revealed that only $21 \%$ of exclusively breast-fed infants had normal weight when compared to bottle-fed infants i.e. $25 \%$. Also, no significant differences were found in the nutritional status of both the groups $(p>0.05)$ as $46 \%$ EBF infants were wasted, $70 \%$ infants were stunted while $44 \%$ were overweight when compared to $\mathrm{BF}$ infants i.e. $44 \%$ and $60 \%$ and $30 \%$, respectively. However, the incidence of allergies, infections (cold and flu) and diarrhea was significantly $(\mathrm{p}<0.05)$ more prevalent in $\mathrm{BF}$ infants when compared to EBF infants.

Conclusion: The study concluded that there are no significant differences in the nutritiona status of exclusively breast-fed and bottle-fed infants. Whereas those infants who are bottle fed for the first six months are more prone to allergies, diarrhea and infections when compared to exclusively breast-fed infants.

Keywords: exclusively breastfeeding, bottle feeding
Volume 6 Issue 4 - 2017

\author{
Zainab Hussain,' Najeeha Khan² \\ 'Rana Liaquat Ali Khan College of Home Economics, Karachi \\ 2Lecturer, Rana Liaquat Ali Khan College of Home Economics, \\ Karachi
}

Correspondence: Zainab Hussain, Rana Liaquat Ali Khan College of Home Economics, Karachi, Pakistan, Email zainabhussain874@gmail.com

Received: December 14, 2016 | Published: March 09, 2017

\section{Background}

Breast milk is considered to be the best and the primary source of nutrition for infants. The World Health Organization (WHO) recommends that infants must be exclusively breast-fed for the first six months. But sometimes due to a number of reasons, infants cannot be breast-fed because their mothers are either sick or suffering from certain chronic diseases or are probably working mothers. One option for infants who are unable to be breast-fed is bottle feed i.e., resorting to powder milk as primary feed for infants. Since bottle feed is the primary source of nutrition for those infants whose mothers cannot breastfeed them, it is important that bottle feed is given appropriately to ensure proper growth and development. ${ }^{1}$

For some parents, or when the baby has certain medical conditions, it is very important to know exactly how much the baby is receiving at each feed, and in such a scenario bottle-feeding allows exact measurement of the feed. Foods, medications, or any physical conditions in the mother are no longer a concern for the baby who is receiving formula. ${ }^{2}$

Bottle feed practices are gaining prevalence in Pakistan as compared to exclusive breast feeding, with the statistics reaching. $41 \%{ }^{3,4}$ In Pakistan, mothers are aware of the advantages and disadvantages of breast and bottle feeding but a disparity is observed between their perception and practices 5. Due to the increasing trend of bottle feeding practices rather than EBF, it is important to highlight the advantages of breastfeeding for those mothers who do not breastfeed, in order to develop in them the inclination to feed their babies unless they are suffering from any chronic condition.

Hence, this study was carried out to assess and compare the nutritional status of exclusively breast fed and bottle fed infants $\leq$ 6months. 


\section{Methods}

This study is a part of larger project titled "ASSESSMENT OF THE NUTRITIONAL STATUS OF BOTTLE-FED INFANTS AND THE PREVALENCE OF INFECTIONS, ALLERGY AND DIARRHEA AMONG BOTTLE FED INFANTS AND ITS COMPARISION WITH EXCLUSIVELY BREAST-FED INFANTS AGED 0-6MONTHS". The research was conducted by a student of BS 8th Semester (2015) of Rana Liaquat College of Home Economics. The current study's aim was to assess the nutritional status of bottle-fed infants and the prevalence of infections, allergy and diarrhea among bottle fed infants aged 0-6months.

The total population of subjects was 100. Data was collected from different hospitals of Karachi by purposive sampling. Tool used to collect data was an interview-based questionnaire which consisted of demographic, anthropometric and general questions to assess the nutritional status of bottle-fed infants.

Interview method was used to collect data to carry out the research. Consent forms were signed from each respondent hospital before the commencement of study. The efficacy of questionnaire was assessed through pre-testing in order to measure the extent of outcomes and pertinent results. Sample size for pre-testing was 10 respondents. A few questions were then rephrased and added.

Each data collector participated equally in the data entry. Data was entered in SPSS Version 17(Statistical Packages for Social Sciences) which was pooled in a single file and cleared. Each student extracted the data for their specific study.

Data was presented using descriptive statistics in the form of frequencies and percentages. Assessment of nutritional status was interpreted in the form of frequency table and bar graphs. Anthropometric measurements were assessed using WHO growth charts.

The characteristics of the subjects are presented in tables and graphs whereas the demographic profile is presented in the form of tables.

\section{Results}

This chapter provides the detailed description of findings of the research on assessment of the nutritional status of bottle-fed infants aged between $0-6$ months. It describes the socio-demographic characteristics of the respondents, infant's feeding practice, anthropometry and the frequency of infections, diarrhea and allergy among bottle fed infants.

\section{Demographic Characteristics of the Subjects}

The sample consisted of 100 infants of which 50 were bottle-fed infants and 50 were exclusively breast-fed infants. Data was collected using a purposive sample technique. Majority of the infants were boys and most infants were 1-2months old. Majority of their mothers were highly educated and belonged to high income family (Table 1).

\section{Anthropometric Characteristics of Bottle Fed Infants}

Infant's weight-for-length, length-for-age, weight-for-length and head circumference-for-age were assessed through WHO growth charts. The results revealed that the growth of most of the bottle fed infants was not up to the standard as head circumference, length for age and weight-for-length of most of bottle-fed infants was below normal. However weight-for-age was good as most BF infants were above normal range. This finding also correlates with the findings of research held in California in which mean weight-for-age of bottle-fed infants remained at or above normal during the first year of infant's life $^{5}$ (Figure 1).

Table I Demographic Characteristics of the Subjects

\begin{tabular}{ll}
\hline Gender & Frequency/percentages \\
\hline Boys & 35 \\
Girls & 15 \\
Age & \\
0-I months & 5 \\
I-2 months & 20 \\
2-3 months & 4 \\
3-4 months & 10 \\
4-5 months & 6 \\
5-6 months & 5 \\
Mother's Education Level \\
Non Educated & 7 \\
<Matriculate & 3 \\
>Matriculate & 5 \\
Graduate & 35 \\
Income Level & \\
>20000 & 8 \\
>50000 & 42 \\
\hline
\end{tabular}

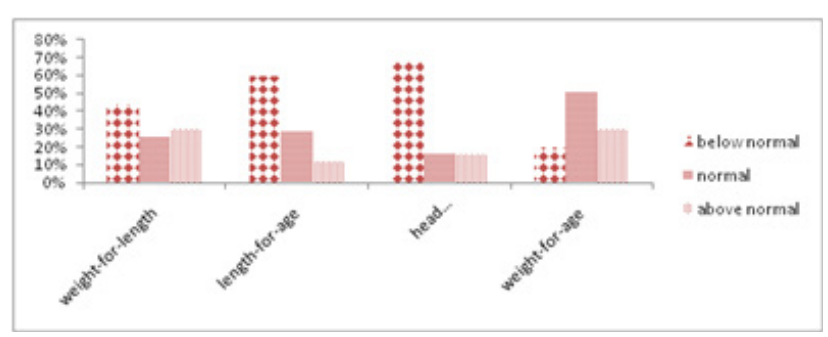

Figure I Anthropometric Characteristics of Bottle-fed infants.

\section{Comparison of Anthropometric Characteristics of Bottle-fed Infants with Exclusively Breast-fed Infants}

It was surprising that majority of the exclusive breast-fed and bottle-fed infants were stunted although they belong to families where mothers were mostly educated i.e. $68 \%$ had mostly 1 or 2 children and belong to high income group. Even at birth most of the infants (44\%) had normal weight. Also, the ratio of stunting and wasting was approximately the same among both groups. In addition, the prevalence of overweight was more among bottle fed infants since early bottle feeding is associated with the risk of obesity rebound, predictive obesity in later life. ${ }^{6}$ But overall no significant differences were found in stunting $(\mathrm{p}=0.138)$, wasting $(\mathrm{p}=0.086)$ and overweight $(p=0.197)$ among both groups of infants (Figure 2).

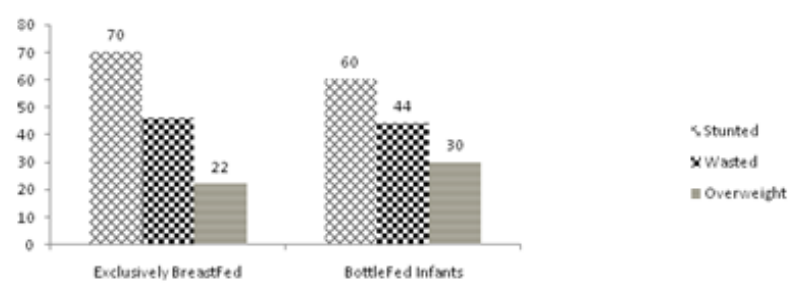

Figure 2 Comparisons of Anthropometric Characteristics of Bottle-fed Infants with Exclusively Breast-fed Infants. 


\section{Frequency of infections, diarrhea and allergy and bottle fed infants}

Majority of the bottle-fed infants never suffered from pneumonia and asthma. However $38 \%$ of bottle-fed infants suffered from cold and flu twice a month, $32 \%$ bottle-fed infants suffered from diarrhea twice a month .Also 16\% bottle-fed infants suffered from allergy twice a month (Figure 3).

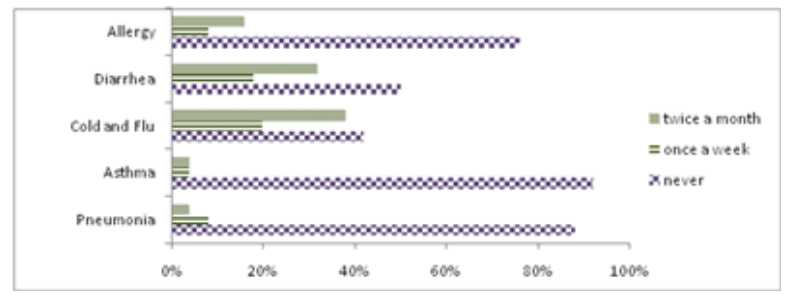

Figure 3 Frequency of infections, diarrhea and allergy among bottle-fed infants.

\section{Comparison of frequency of infections, diarrhea and allergy among exclusively breast-fed and bottle-fed infants}

The results revealed that no significant differences were found in the frequency of asthma $(p=0.264)$ and pneumonia $(p=0.10)$ in most of the exclusively breast-fed and bottle-fed infants. But, the frequency of cold and flu was more prevalent in bottle fed infants with a significant difference $(\mathrm{p}=0.05)$. Also, there were no significant differences $(p=0.254)$ in the frequency of diarrhea among both groups of infants. In addition, the frequency of allergy was also prevalent in bottle fed infants with a significant difference $(\mathrm{p}=0.001)$. This correlates with a cohort study in Sweden where exclusive breast feeding was associated with a preventive effect of on the early development of allergic diseases $^{7}$ (Figure 4).

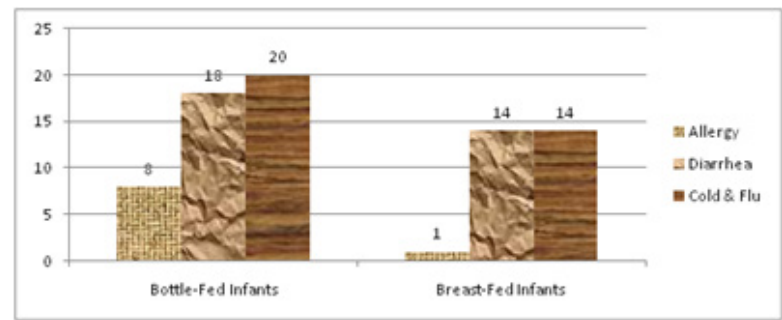

Figure 4 Comparison of frequency of infections, diarrhea and allergy among exclusively breast-fed and bottle-fed infants.

\section{Frequency of stools of Bottle-fed Infants}

$72 \%$ of the bottle-fed infants passed $<3$ stools per day and $28 \%$ infants passed equal to or greater than 3 stools per day (Figure 5).

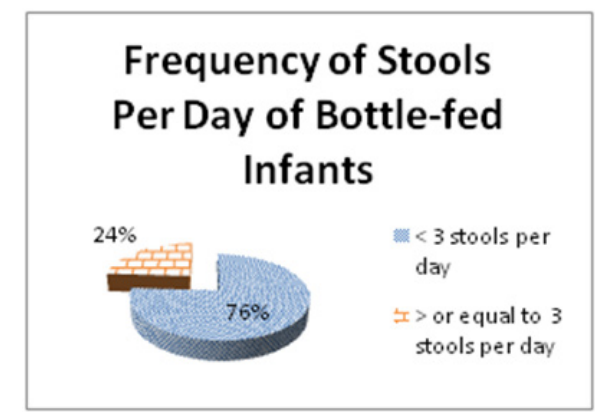

Figure 5 Frequency of stools of Bottle-fed Infants.

\section{Comparison of Frequency of stools of bottle fed infants with exclusively breast-fed infants}

According to the results, diarrhea was more common in BF infants than in EBF infants as it shows that in BF infants $26 \%$ were suffering from diarrhea whereas $24 \% \mathrm{EBF}$ infants were suffering from diarrhea with a significant difference $(\mathrm{p}=0.024)$ (Figure 6).

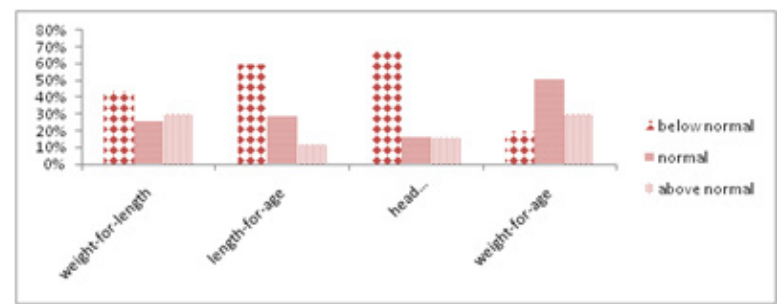

Figure 6 Comparison of Frequency of stools of bottle fed infants with exclusively breast-fed infants.

\section{Age of the Infant When Bottle-feeding was started}

The results showed that mostly mothers started bottle-feeding their child at a very early age i.e. 0-1 month (Figure 7).

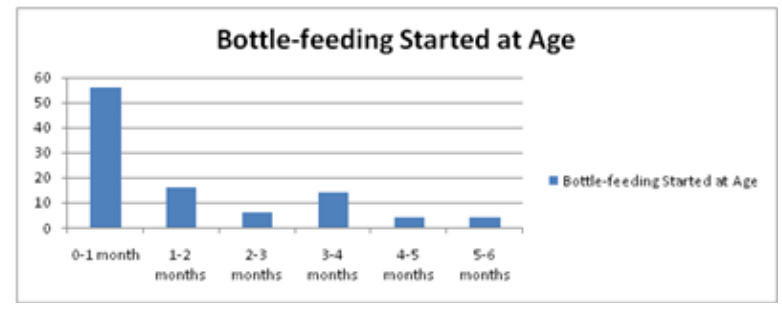

Figure 7 Age of the Infant at which Bottle-feeding was introduced.

\section{Reasons for Adoption of Bottle-feeding}

The reasons for adopting bottle-feeding for infants included that mostly mothers were working mothers (46\%) while $40 \%$ mothers complaint of less production of milk and only $14 \%$ mothers had other reasons including some kind of maternal disease, next pregnancy, social taboos like baby not gaining weight, or the presence of twins. A research was also conducted in United States for knowing the reasons for adoption of bottle-feeding which correlates with this research i.e. mostly mothers had to return to work soon after the delivery and some of them were uncertain regarding the quantity of breast milk (Figure 8).

The study assessed that the overall nutritional status of bottle-fed infants was far much better than exclusively breast-fed infants. One possible reason could be that mostly mothers were educated and belong to middle income families and because they had proper knowledge of bottle feeding practices, their infants had good nutritional status. It was seen that socio-demographic characteristics of mothers have significant influence on feeding practices.

\section{Discussion}

\section{Importance of bottle feeding for infant's health}

It is important to remember that breastfeeding is the best and most natural source of nutrition for the baby. The World Health Organization and the Department of Health recommend that the baby should be breastfed exclusively for the first six months and the practice should be continued even with the introduction of weaning foods. This is the safest and the best way to protect the baby's health. 
If a mother chooses not to breastfeed, it is important that she always prepares the baby's bottle feeds properly. On switching from breastfeeding or introducing formula feeds for the first time, public health nurse's advice must be sought. ${ }^{8}$

Bottle feeding is an important topic because conventional bottle feeding methods can cause problems for both breastfed and bottle fed babies, and all babies deserve to be fed in a proper and hygienic manner. ${ }^{9}$

\section{Bottle feeding}

It requires the infant receives any liquid (including breast milk) per semi-solid food with a nipple/teat. ${ }^{10}$

\section{Exclusive breastfeeding}

It requires that the infant receives breast milk (including milk expressed or from a wet nurse). It may also allow the infant to receive ORS, drops, syrups (vitamins, minerals and medicines). ${ }^{10}$

\section{Breast feeding}

It requires that the infant receives breast milk (including milk expressed or from a wet nurse). It may also allow the infant to receive any food or liquid including non-human milk and formula. ${ }^{10}$

\section{Exclusive breast feeding v/s bottle feeding practices in Pakistan}

According to estimates of UNICEF, exclusive breastfeeding rate in Pakistan was 37.1 percent in 2000-01, which was the lowest among all South Asian countries. ${ }^{3}$

According to the Demographic Health Survey, this percentage has risen only from 37.1 per cent in 2006-07 to 37.7 per cent in 2012-13. However, when it comes to the bottle-feeding practice, Pakistan has no close competitors; bottle-feeding rates have risen from an already undesirable 32.1 per cent in 2006-07 to 41 per cent in 2012-13.

\section{Reasons for adopting of bottle-feeding practices}

It has been observed that the most common reasons of adopting bottle-feeding practices are inadequate milk production $(71 \%)$, maternal employment $(11.2 \%)$, social taboos like baby not gaining weight $(5.2 \%)$, loose stools or constipation (3.3\%), maternal systemic illness $(3.6 \%)$, and the presence of twins $(1.5 \%) .{ }^{11}$

\section{Inconvenience while breastfeeding}

- Having a baby can be an extremely tiring for everyone involved, and mostly mothers. Bottle feeding facilitates especially the mother in feeding the baby. Adopting bottle-feeding practices prove helpful especially at night-time, allowing the mother to get a few hours of uninterrupted sleep.

- Bottle feeding may allow the partner to develop a closer bond with the baby.

- If a woman has to go back to work or go somewhere without her baby, breastfeeding can become more difficult.

- However, some women may find breast-feeding more convenient because they don't have to carry bottles or equipment when they go anywhere, while others find it quite difficult to breast-feed in certain places, for example in a restaurant. The discomfort of breast-feeding in public places necessitates bottle feeding occasionally. ${ }^{8}$
- Moreover, mothers are often made to feel 'marginalized and ashamed' when they breastfeed in public, according to an international study.

A common theme of the study was the shame felt by mothers who breastfeed their babies. For example, some breastfeeding mothers discussed concerns about how they are viewed by others when exposing their bodies in public.

Breastfeeding: not as easy as it looks: There are some demerits of breastfeeding due to which mothers are likely to give bottle-feed to their child as breastfeeding may cause sexual issues or may cause breast pain or differences in breast size. ${ }^{12}$

\section{Merits of bottle feeding practices}

- Bottle feeding is usually easier for mothers and babies to learn.

- It's comforting to see how much the baby is taking at each feed.

- Any trusted person of your choice can feed the baby.

- New mothers may take a baby-feeding break and get much needed rest.

- Bottle feeding in public is easily accepted.

- Mother's who bottle-feed may take medications as needed without worry of passing unwanted chemicals to their baby. ${ }^{13}$

\section{Demerits of bottle-feeding practices}

Although there are many merits of bottle-feeding, but when it comes to the health of an infant, bottle-feeding has many demerits as well. Health outcomes in developed countries differ substantially for mothers and infants who give bottle feed compared with those who breastfeed. For infants not being breastfed is associated with an increased incidence of infectious morbidity, as well as elevated risks of childhood obesity, type 1 and type 2 diabetes, leukemia, and sudden infant death syndrome (SIDS), pneumonia, constipation, etc. For mothers, failure to breastfeed is associated with an increased incidence of premenopausal breast cancer, ovarian cancer, retained gestational weight gain, type 2 diabetes, myocardial infarction, and the metabolic syndrome. ${ }^{14}$

Bottle feeding and the risk of obesity in later life: In recent years a link between formula feeding of infants, and childhood and adult obesity has been established. This link between has been hotly debated with researchers making staunch claims on both sides. However, the fact remains that when examining the general population, adults who were breastfed are less likely to be overweight or obese than those who were formula fed as infants. ${ }^{15}$

This was proved in a follow up study of children up to 6years of age where it was revealed that by 3 months, bottle-fed infants had significantly higher BMIs and thicker skin folds than breastfed infants. From 6months onwards, compared to breastfed children, a consistently higher proportion of bottle-fed children exceeded the 90th and the 97th percentile of BMI and skin-fold thickness reference values. From the age of 4 y to 5 and $6 y$, in bottle- fed children, the prevalence of obesity nearly doubled and tripled, respectively. ${ }^{16}$

Bottle feeding relates to Infectious Morbidity: Compared with breastfed infants, formula-fed infants face higher risks of infectious morbidity in the first year of life. These differences in health outcomes can be explained, in part, by specific and innate immune factors present in human milk. Plasma cells in the mother's bronchial tree and intestine migrate to the mammary epithelium and produce $\operatorname{IgA}$ 
antibodies specific to antigens in the mother-infant dyad's immediate surroundings, providing specific protection against pathogens in the mother's environment. In addition, innate immune factors in milk provide protection against infection. ${ }^{16}$

Bottle feeding relates to Otitis Media: Approximately $44 \%$ of infants will have at least 1 episode of otitis media in the first year of life, and the risk among formula-fed infants is doubled $(95 \%$ confidence interval compared with infants who are exclusively breastfed for more than 3months). Human milk oligosaccharides and antibodies to common respiratory pathogens in the infant's environment are thought to provide protection from infection. ${ }^{16}$

Bottle feeding and risks of lower respiratory tract infections: Infants who were not breastfed face increased risk $(95 \%)$ of hospitalization for lower respiratory tract infection in the first year of life, compared with infants who were exclusively breastfed for more than 4 months. Therefore, breastfeeding should be encouraged because lipids in human milk appear to have antiviral activity against respiratory tract infections. ${ }^{16}$

Bottle feeding and risks of gastrointestinal infections: Bottle fed infants face an increased risk of gastroenteritis and diarrhea. Infants who are bottle fed are 2.8times more likely to develop gastrointestinal (GI) infection than those who are exclusively breastfed. ${ }^{16}$

Neurodevelopment: Multiple authors have examined associations between infant feeding and cognitive development, with mixed results. Several studies reported modestly lower IQ scores in bottle fed children compared with breastfed children, whereas others reported no association between infant feeding and intelligence. ${ }^{16}$

Risks of Sudden Infant death Syndrome (SIDS): It is seen that formula feeding is associated with increased risk of SIDS compared with breastfeeding. These associations persisted after adjustment for sleeping position, maternal smoking, and socioeconomic status. ${ }^{16}$

Infant Mortality: After adjusting for maternal age, education, smoking status, infant race, gender, birth weight, congenital malformation, and birth order, bottlefeedinginfantsare at higher risk of infant mortality compared with breastfeed infants. ${ }^{16}$

Bottle feeding and risks of Pneumonia: Pneumonia is the major cause of death of children younger than 5years of age worldwide, accounting for 4.3 million deaths per year, or $>10,000$ per day. Pneumonia is currently the leading cause of morbidity, hospitalization, and death in children under-five. Children who were not breast-fed were found to be more prone to develop severe pneumonia (78.1\%) than those who were breastfed (54.4\%). ${ }^{17}$

Bottle-feeding and Diarrhea: Diarrhea can usually be defined as an increase in stool frequency to twice the usual number per day in infants. ${ }^{18}$ Bottle-fed infants, compared with the exclusively breastfed ones, have a higher incidence of diarrhea because of not sterilizing bottles properly. Exclusively breast-fed infants, as they receive mother milk are less prone to diarrhea because of the presence of several antiinfective factors in human milk. Oligosaccharides, contained in the human milk, are one of the important defensive factors against acute diarrheal infections in exclusively breast-fed infants. ${ }^{17}$

\section{Conclusion}

It was concluded that there are no significant differences $(p>0.05)$ between the nutritional status of exclusively breast fed and bottle fed infants. However, the incidence of allergies, infections (cold \& flu) and diarrhea is more prevalent $(\mathrm{p}<0.05)$ in bottle-fed infants as compared to exclusively breast-fed infants. But there is a need to assess in future researches about how to reduce the prevalence of allergies and diarrhea in bottle-fed infants as these can have a negative impact on infant's health.

\section{Acknowledgements}

First and foremost, I am grateful to Almighty ALLAH for giving me endurance to this research, for helping me in every single step of this research study and making it possible. I am thankful to my advisor Miss Najeeha Khan whose expertise, understanding and generous guidance and moral support enabled me to complete my research without any difficulty.

It won't be fair if I don't express my thanks to Miss Gul e Erum who took out her precious time to edit this report and bring in more clarity.

Last but not the least; I would like to express my gratitude to my beloved family who in one way or another extended their unconditional support.

\section{Conflicts of interest}

Author declares there are no conflicts of interest.

\section{Funding}

None.

\section{References}

1. Ali Sumera, Ali Faizan Syed, Imam Malick Ayesha, et al. Perception and practices of breast feeding of infants 0-6 months in an urban and semiurban community in Pakistan a cross sectional study. J Pak Med Assoc. 2011;61(1):99-104.

2. Alison Stuebe. The Risks of Not Breastfeeding for Mothers and Infants. Rev Obstet Gynecol Fall . 2009;2(4):222-223.

3. Alison Stuebe. The Risks of Not Breastfeeding for Mothers and Infants. Rev Obstet Gynecol Fall. 2009;2(4):222-231.

4. http://patch.com/california/paloalto/benefits-of-bottle-feeding/

5. K E Bergmann, R L Bergmann, R Von Kries, et al. Early determinants of childhood overweight and adiposity in a birth cohort study. Int J Obes Relat Metab Disord . 2003;27(2):162-172.

6. J Surg Pak. Factors associated with failure of exclusive breast feeding 2006;11(1):24-26.

7. Safari JG, Kimambo SC, Lwelamira JE. Feeding practices and nutritional status of infants in Morogoro Municipality. Tanzan J Health Res . 2013;15(3):178-185.

8. Ibrahim S, Ansari NS. Factors associated with failure of exclusive breast feeding. J Surg Pak. 2006;11(1):24-26.

9. http://www.irishhealth.com/article.html?con=62

10. http://www.pregnancy.com.au/breastfeeding/breastfeeding information/formula-feeding-and-childhood-obesity-is-there-a-l.shtml

11. Bergmann KE, Bergmann RL, Von Kries R, et al. Early determinants of childhood Overweight and adiposity in a birth cohort study. Int $J$ Obes Relat Metab Disord. 2003;27(2):162-172.

12. http://www.medicinenet.com/breastfeeding/page3.html

13. http://www.livestrong.com/article/492850-disadvantages-ofbreastfeeding/

14. http://www.thenews.com.pk/Todays-News-6-199241-Pakistan-makeszero-progress-in-exclusive-breastfeeding-in-seven-years

15. Merlin- Nowshera.United Nation International Children's Monitory 
Fund. Global Breast Feeding Week Celebration. 2011.

16. World Health Organization. Indicators for assessing infant and young child feeding practices. WHO Press. Washington D.C.WHO Publications. 1990.

17. http://www.who.int/mediacentre/ factsheets / fs330/en/
18. http://pediatrics.aappublications.org/content/139/3?current-issue $=y$

19. KE Bergmann, R L Bergmann, RVon Kries, et al. Early determinants of childhood overweight and adiposity in a birth cohort study. Int $J$ Obes Relat Metab Disord . 2003;27(2):162-172. 